\title{
Control-oriented modelling and simulation of a variable-frequency efficiency-optimized flyback converter
}

\author{
Tue T. Vu ${ }^{1}$, Seamus O’Driscoll ${ }^{2}$ and John V. Ringwood ${ }^{1}$
}

\begin{abstract}
Driven by the requirement for a fairly accurate and fast simulation of a variable frequency digital controlled flyback converter, this paper develops a control-oriented mathematical model, which retains the essential dynamic characteristics of the real system, but offers significant computational complexity reduction compared to a full modelling approach. In addition to the model simplification, an approach to further improve the simulation speed, through a specific implementation of the differential equation solver, is also considered. To verify the accuracy of the proposed model, different tests with both open-loop and closed-loop flyback converters are performed. The results from both simulation and experiment show good agreement in all the test cases.
\end{abstract}

\section{INTRODUCTION}

Driven by the new energy standards, i.e. U.S. Energy Star [1], and market demand for external AC/DC power supplies, the control solution for flyback converters is gradually moving toward a digital design with multi-feature integration [2]-[4]. The performance and stability of some functions, such as primary side sensing (PSS), $\mathrm{n}^{\text {th }}$ valley switching operation (VSO), efficiency optimization (EO), can not be simply verified by an averaged-small-signal model or an ideal switched converter model. So a detailed simulation, including the effects of parasitic components, is required. In addition to the minimum accuracy requirement, a fast execution speed is also necessary to handle the large disparity in the converter dynamics, including very fast events due to switching devices, and much slower events arising from input voltage, output load, and operating mode transients. Therefore, a fairly accurate and fast simulation of a variable frequency flyback converter, as shown in Fig. 1 is a problem of considerable interest.

Emulating the behaviour of the open-loop flyback converter in Fig. 1 can be achieved by either a full modelling approach [5], i.e. with detailed complex models for circuit devices, or a simplified one, i.e. with simple idealized device models [6]. The differences between these two approaches are essentially the accuracy and computational complexity of the models employed. The full model in [5] can provide a very high level of accuracy and insight, however, its computational complexity is too great to permit its use as a basis for digital control design. On the other hand, the

\footnotetext{
*This work was supported by the Semiconductor Research Corporation (SRC), Dallas, under Contract 2008-HC-1836

${ }^{1}$ Tue T. $\mathrm{Vu}$ and John V. Ringwood are with Electrical Engineering Department, National University of Ireland, Maynooth, Ireland ttrongvu@eeng.nuim.ie, john.ringwood@eeng. nuim.ie

${ }^{2}$ Seamus O'Driscoll is with Texas Instrument Ltd., Cork, Ireland seamusodriscolldti.com
}

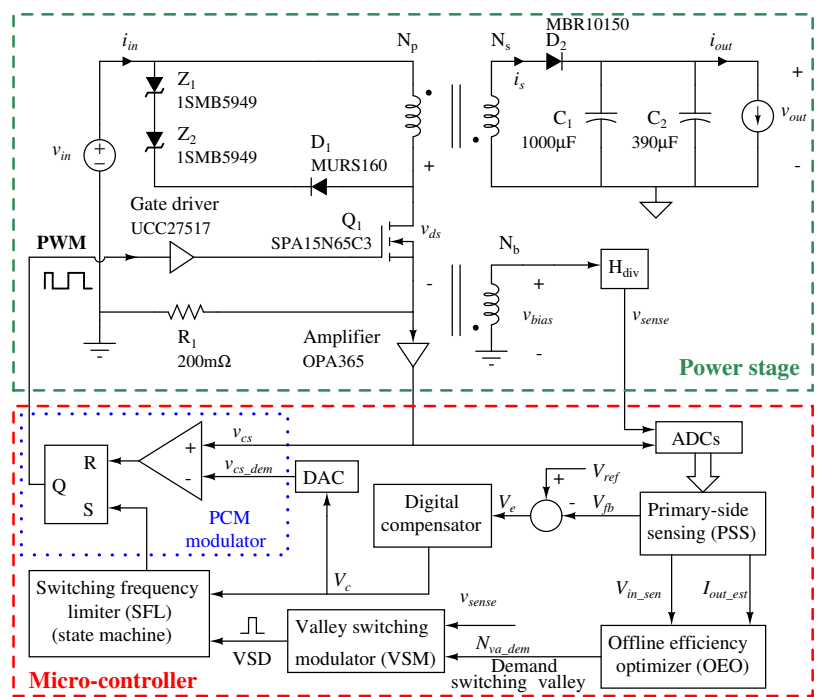

Fig. 1. Circuit diagram of digital peak current mode (PCM) controller with primary-side sensing (PSS) and off-line efficiency optimization (OEO) for a flyback converter application

idealized model in [6] ignores most dynamics occurring within switching cycles, and consequently is not appropriate for control design either. Therefore, we require to develop a control-oriented model which, ideally, retains the essential dynamic characteristics of flyback converters, but offers significantly reduced complexity. Such an objective is the focus of this paper.

The simulation of a converter model can be performed by either deriving and solving a set of differential equations using general equation-solver programs, such as MATLAB/SIMULINK, or by directly employing a circuit-oriented simulator, like PSIM. Although the first method requires extra effort to prepare the equations and set up the solver, it offers full control of the solver configuration, physical insight into the operation of the system, and so is employed in this study.

\section{CONTROL-ORIENTED MODEL OF FLYBACK CONVERTERS}

The execution time of the simulation and the modelling accuracy are strongly affected by the complexity of the model of the converter circuit, particularly for the transformer and semiconductor devices. For example, an idealized model needs only a short time to complete the simulation of the flyback converter over many switching cycles; however, the data generated by such an idealized model is mostly too poor, 
in terms of information content, and is not adequate for the design of the digital control in Fig. 1. Conversely, increasing model complexity allows the reproduction of all transient details in each switching cycle, but the simulation task may consume a significant amount of time if a full transient event, e.g. step load response, is of interest. It is obvious that the application of the full converter model to control design is technically impractical; however, the full model can be used as a basis to obtain a control-oriented model. In particular, based on the full model, we can decide which parts of it need to be simplified and which need to be preserved in order to satisfy the simulation time and accuracy

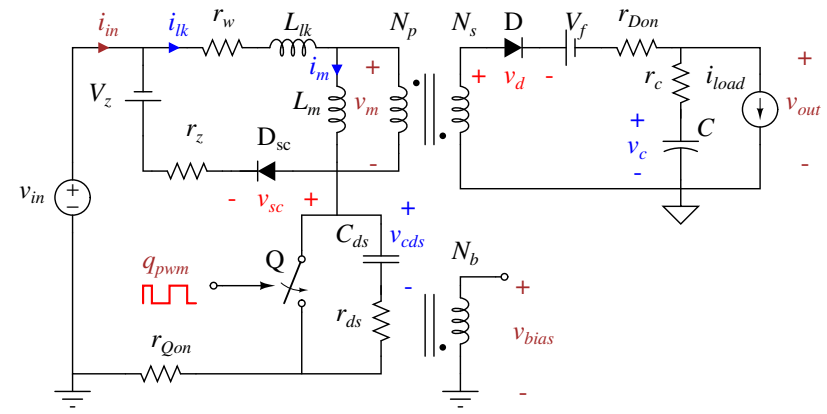

Fig. 2. Control-oriented model of a flyback converter. Q is an ideal switch controlled by the PWM signal. Diodes $\mathrm{D}$ and $\mathrm{D}_{\mathrm{s}}$ have ideal characteristics which are zero resistance when on and open circuit when off.

In general, reducing model complexity results in losing information fidelity in the simplified model. Depending on the modelling purposes, some information is critical for control design process and needs to be preserved, while other information can be eliminated. For example, $\mathrm{n}^{\text {th }}$ valley switching control in [2]-[4] needs certain information from the feedback signals, including the oscillation in $v_{\text {bias }}$ during discontinuous conduction mode (DCM), and the high frequency ringing in $v_{\text {bias }}(t)$ after the MOSFET turn-off instance, in order to make a correct decision. Without such information, the model should not be used for the control design process. Since the information contained inside the model itself is far more important than the complexity of the model, model simplification should focus on the impact of information lost rather than a pure focus on complexity. Ideally, we want to maximize complexity reduction for minimum impact on accuracy.

The flyback converter model obtained after a simplification step is sketched in Fig. 2, where the transformer is described by a traditional $\mathrm{T}$-model with a leakage inductance $L_{l k}$ and magnetizing inductance $L_{m}$, while the MOSTFET is modelled by an ideal MOSFET $Q$ in parallel with a parasitic RC circuit. The leakage inductance $L_{l k}$ and capacitance $C_{d s}$ are utilized in Fig. 2 to reproduce all the ringing in the bias winding voltage $v_{\text {bias }}(t)$, which is critical for control performance assessment. The Zener diodes, $\mathrm{Z}_{1}$ and $\mathrm{Z}_{2}$ in Fig. 1, are simply represented by a voltage source $V_{z}$ in series with $r_{z}$ in Fig. 2, while the Schottky diodes, $\mathrm{D}_{1}$ and $\mathrm{D}_{2}$ in Fig. 1, are modelled by ideal diodes $\mathrm{D}_{\mathrm{sc}}$ and $\mathrm{D}$ in Fig. 2, respectively. To represent the conduction losses occurring in the MOSFET, transformer and diode, three resistors $r_{Q o n}, r_{w}$, and $r_{D o n}$ are added to the control-oriented model in Fig. 2.

TABLE I

STATE TRANSITION CONDITIONS FOR SWITCHING DEVICES.

\begin{tabular}{|c|c|c|}
\hline Devices & State transition & Boundary condition \\
\hline \multirow{2}{*}{$\mathrm{Q}$} & off $\rightarrow$ on & $q_{p w m}(t)=1$ \\
\cline { 2 - 3 } & on $\rightarrow$ off & $q_{p w m}(t)<1$ \\
\hline \multirow{2}{*}{$\mathrm{D}$} & off $\rightarrow$ on & $i_{d}(t)=0$ and $v_{d}(t)>0$ \\
\cline { 2 - 3 } & on $\rightarrow$ off & $v_{d}(t)=0$ and $i_{d}(t)<0$ \\
\hline \multirow{2}{*}{$\mathrm{D}_{\mathrm{sc}}$} & off $\rightarrow$ on & $i_{s c}(t)=0$ and $v_{s c}(t)>0$ \\
\cline { 2 - 3 } & on $\rightarrow$ off & $v_{s c}(t)=0$ and $i_{s c}(t)<0$ \\
\hline
\end{tabular}

The operation of the switch $\mathrm{Q}$ in Fig. 2 is externally controlled by the PWM signal $q_{p w m}(t)$, while the transition between the on- and off-states of the diodes $\mathrm{D}$ and $\mathrm{D}_{\mathrm{sc}}$ is not directly controlled by external control action but rather occurs when the internal states, including diode terminal voltages and currents, reach particular boundaries or threshold conditions. The boundary conditions for the state transitions of the three semiconductor devices are summarized in Table I. Notice that all $\mathrm{Q}, \mathrm{D}$, and $\mathrm{D}_{\mathrm{sc}}$ are equivalent to zero resistance when on, and open circuit when off.

Since the circuit diagram in Fig. 2 contains only ideal switches and linear circuit components, it can be described by a piecewise linear time invariant (LTI) model, whose operation over a period, when the switch positions are frozen, is governed by

$$
\begin{aligned}
\frac{d \mathbf{x}(t)}{d t} & =\mathbf{A}_{i} \mathbf{x}(t)+\mathbf{B}_{i} \mathbf{u}(t) \\
\mathbf{y}(t) & =\mathbf{C}_{i} \mathbf{x}(t)+\mathbf{D}_{i} \mathbf{u}(t)
\end{aligned} \quad i=1,2, \ldots
$$

where

$$
\mathbf{x}(t)=\left[\begin{array}{c}
i_{l k}(t) \\
i_{m}(t) \\
v_{c d s}(t) \\
v_{c}(t)
\end{array}\right], \quad \mathbf{y}(t)=\left[\begin{array}{c}
i_{\text {in }}(t) \\
v_{m}(t) \\
v_{\text {out }}(t)
\end{array}\right], \quad \mathbf{u}(t)=\left[\begin{array}{c}
v_{\text {in }}(t) \\
i_{\text {load }}(t) \\
V_{f} \\
V_{z}
\end{array}\right] .
$$

$\mathbf{x}(t), \mathbf{y}(t)$ and $\mathbf{u}(t)$ are the state, output and input variables of the converter model, respectively while $\mathbf{A}_{i}, \mathbf{B}_{i}, \mathbf{C}_{i}$ and $\mathbf{D}_{i}$ denote the state space matrices for a given combination of the three switch positions of $\mathrm{Q}, \mathrm{D}$ and $\mathrm{D}_{\mathrm{sc}}$. In theory, 8 possible circuit configurations can be formed from 3 independent switches. However, only 5 combinations are feasible in the control-oriented model in Fig. 2, the other 3 configurations do not exist because $\mathrm{D}$ and $\mathrm{D}_{\mathrm{sc}}$ are only 2-terminal semiconductors, whose states are controlled internally by the system states. The expressions for the state space matrices for the 5 switch configurations are presented in the Appendix.

Given the external excitation $v_{\text {in }}(t), i_{\text {load }}(t)$ and the PWM control signal $q_{p w m}(t)$, the voltage and current signals in Fig. 2 can be obtained by solving the set of LTI ordinary differential equations (ODEs), in Eq. (1), over time. The main challenge is the sequence in which the differential equations are solved, which is unknown in advance and depends on the 
states of the switch Q, and diodes D and $\mathrm{D}_{\text {sc }}$. Fortunately, switching from one topology to another in the converter circuit can be simply determined through the transition in the states of the semiconductor devices, particularly through the transition in the values of $q_{p w m}(t), i_{d}(t), v_{d}(t), i_{s c}(t)$ and $v_{s c}(t)$; refer to Table I for more detail. The terminal voltage and current signals $i_{d}(t), v_{d}(t), i_{s c}(t)$ and $v_{s c}(t)$ can be derived from the state variable via

$$
\mathbf{y}_{d}=\mathbf{E}_{i} \mathbf{x}(t)+\mathbf{F}_{i} \mathbf{u}(t) \quad i=1,2, \ldots
$$

where $\mathbf{y}_{d}=\left[i_{d}(t) v_{d}(t) i_{s c}(t) v_{s c}(t)\right]^{T}$, and $\mathbf{E}_{i}, \mathbf{F}_{i}$, are specific matrices for each combination of $\mathrm{Q}, \mathrm{D}$, and $\mathrm{D}_{\mathrm{sc}}$. Due to limited space, the detailed formulae of these matrices are not included in this paper, but one can easily derive them from the diagram in Fig. 2.

\section{MODEL IMPLEMENTATION AND CONVERGENCE HANDLING}

Given the mathematical description of the simplified model in Section II, a differential-equation solver is required to calculate the converter state and output variables for a given excitation condition. The calculation time is typically not fixed and depends on how the solver is designed and the level of accuracy required [6]. By combining the conditions in Table I and Eqs. (1) and (2) together, one can realize that the operation of the control-oriented model is generally governed by differential algebraic equations (DAEs) rather than pure ordinary differential equations (ODEs). Since DAEs contain algebraic relations among state variables, input variables and output variables, a very fine time-step is typically utilized to ensure the convergence of the solution. Such a time-step will lead to a slow execution speed and a long simulation time even for a short transient event. In order to perform faster simulations, it is essential to remove the algebraic loops and convert the DAEs to pure ODEs.

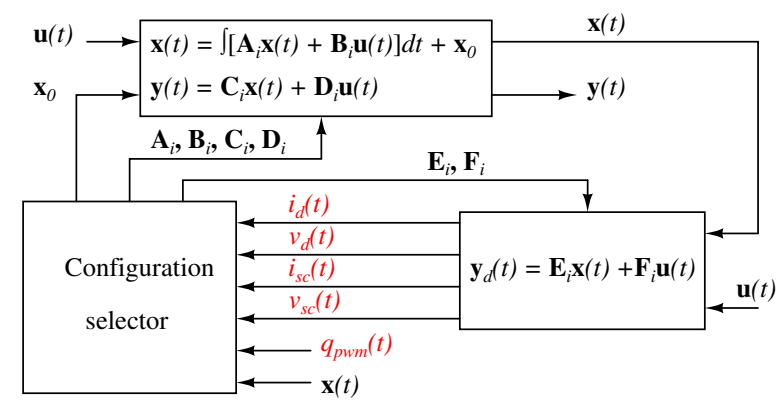

Fig. 3. General approach to simulate the LTI piecewise model in Fig. 2. Basically, the procedure includes three sequential steps: (1) solving the ordinary differential equation which describing the converter operation for a given switch configuration, (2) calculating the state transition signals, and (3) choosing the correct configuration and updating the equation parameters for the next phase.

Breaking the algebraic relations in Eqs. (1) and (2) and Table I can be achieved by sequencing these model equations and forcing an ending condition to avoid re-iteration. An equation-solving sequence, designed for the control-oriented model, is illustrated in Fig. 3. The principle of the procedure in Fig. 3 can be explained as follow. For each time step, the ODE in Eq. (1) is solved using a numerical integration algorithm. The results from the equation solver are utilized to calculate the diode currents and voltages $i_{d}(t), v_{d}, i_{s c}(t)$, and $v_{s c}(t)$. Based on these signals and the PWM signal $q_{p w m}(t)$, the configuration selector chooses the next circuit topology according to the conditions in Table I and then updates the equation parameters in the next time step.

The simulation of the simplified model in Fig. 2 consists of dividing the time duration into smaller time steps and calling the procedure in Fig. 3 at each time step. The selection of the time step can be fixed or variable in size. In this study, a fixed time step is preferred because it allows the implementation and verification of digital control functions. In addition to the algebraic loop removal, the choice and implementation of the ODE solver also affects the simulation speed and accuracy [6]. For simplicity, this paper chooses the Explicit $4^{\text {th }}$-order Runge-Kutta method [7] to obtain the solution of Eq. (1), but other numerical integration methods are also applicable.

\section{OPEN-LOOP SIMULATION AND EVALUATION}

Though the procedure in Section III can be implemented in any numerical computing program, the MAT$\mathrm{LAB} / \mathrm{SIMULINK}$ environment is chosen in order to reduce the development time and to take advantage of our existing control design. The parameter values of the proposed simplified model, which are shown in Table II, are derived based on the data-sheets of the circuit components in Fig. 1 and the previously determined converter model in [5].

TABLE II

PARAMETER VALUES OF THE CONTROL-ORIENTED MODEL

\begin{tabular}{|c|c|}
\hline Input voltage $v_{\text {in }}(t)$ & $120 \mathrm{~V}-373 \mathrm{~V}$ \\
\hline Output voltage $V_{\text {out }}$ & $19.5 \mathrm{~V}$ \\
\hline Magnetizing inductance $L_{m}$ & $791.76 \mu \mathrm{H}$ \\
\hline Leakage inductance $L_{l k}$ & $8.03 \mu \mathrm{H}$ \\
\hline Winding resistance $r_{w}$ & $0.4 \Omega$ \\
\hline Winding turns $N_{p}: N_{s}: N_{b}$ & $46: 10: 6$ \\
\hline Output capacitor $C$ & $900 \mu \mathrm{F}$ \\
\hline Capacitor resistance $r_{c}$ & $10 \mathrm{~m} \Omega$ \\
\hline Forward voltage drop $V_{f}$ & $0.45 \mathrm{~V}$ \\
\hline Resistance, $r_{\text {Don }}, r_{Q o n}, r_{d s}$ & $0.05 \Omega, 0.4 \Omega, 50 \Omega$ \\
\hline Zener voltage $V_{z}$ and resistance $r_{z}$ & $180 \mathrm{~V}, 0.5 \Omega$ \\
\hline
\end{tabular}

Evaluating the accuracy of the intra-cycle responses of the control-oriented model can be achieved by comparing the simulated results, based on the previously determined model in [5] and the proposed model in Section II. For comparison purposes, the working conditions of the flyback converter, which have been examined in [5], are reconsidered here. In particular, the operating condition is chosen as $R=16.829 \Omega$, $v_{\text {in }}=150 \mathrm{~V}, d=0.38$, and $f_{p w m}=50 \mathrm{kHz}$. Such a condition forces the flyback converter to stay in DCM and also forces 

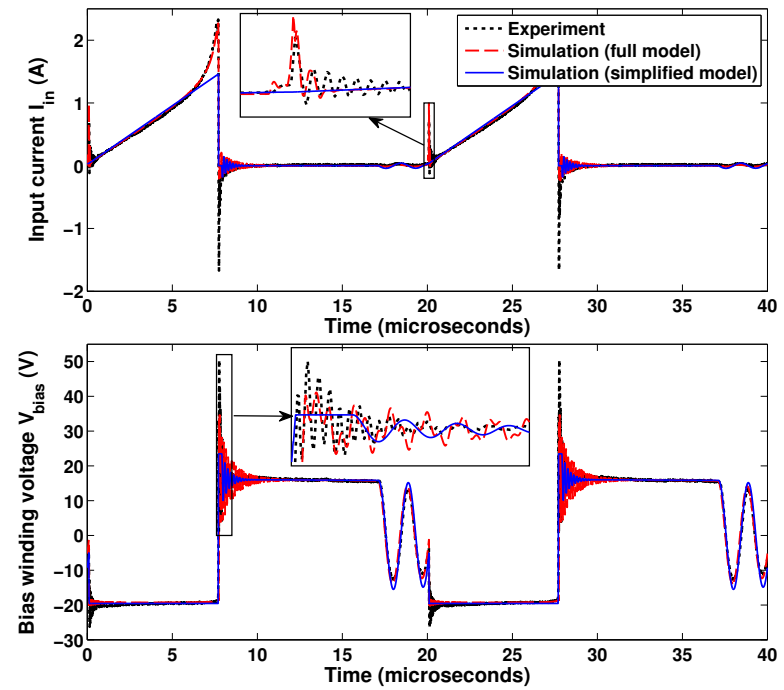

Fig. 4. Converter waveforms obtained from hardware prototype, full and simplified models. The operating point is chosen as the load resistor $R=$ $\frac{v_{\text {out }}}{i_{\text {out }}}=16.829 \Omega, v_{\text {in }}=150 \mathrm{~V}$, duty ratio $\mathrm{d}=0.38, f_{p w m}=50 \mathrm{kHz}$

TABLE III

COMPUTATIONAL COMPLEXITY OF THE FULL AND CONTROL-ORIENTED MODELLING STRATEGIES

\begin{tabular}{|c|c|c|}
\hline \multirow{2}{*}{$\begin{array}{c}\text { Duration of hardware test } \\
\text { (real-time) }\end{array}$} & \multicolumn{2}{|c|}{ Execution time for simulations } \\
\cline { 2 - 3 } & $\begin{array}{c}\text { Full model } \\
\left(19^{\text {th }} \text { order }\right)\end{array}$ & $\begin{array}{c}\text { Simplified model } \\
\left(4^{\text {th }} \text { order }\right)\end{array}$ \\
\hline 50 milliseconds & 17.238 hours & 99.252 seconds \\
\hline
\end{tabular}

the transformer to operate around the saturation region of the ferrite core. The simulated results obtained from the simplified model and the full model are plotted in Fig. 4. In addition to the simulated results, the experimental results are also added to Fig. 4, for comparison purposes.

In order to evaluate the computational complexity of the control-oriented model, the total execution time for a simulation based on the full $19^{\text {th }}$ order model and the proposed $4^{\text {th }}$ order model are computed and listed in Table III. The data from Table III shows that using the control-oriented model allows us to reduce the execution time of the simulation by around 3 orders of magnitude, compared with the full model.

Examining the experimental and simulated waveforms in Figs. 4, and the data in Table III, reveals that the full model is capable of accurately reproducing all the non-linear and high-frequency transient responses at the expense of a huge computation time. In contrast to the full model, the controloriented model is unable to predict all non-linearity or highfrequency dynamics, but requires a significantly shorter time to finish the same job and, most importantly, can retain the fidelity of important information, e.g. ringing in $v_{\text {bias }}(t)$, required by control design needs.

Since the purpose of the proposed model is control design, the accuracy of the inter-cycle responses is as important

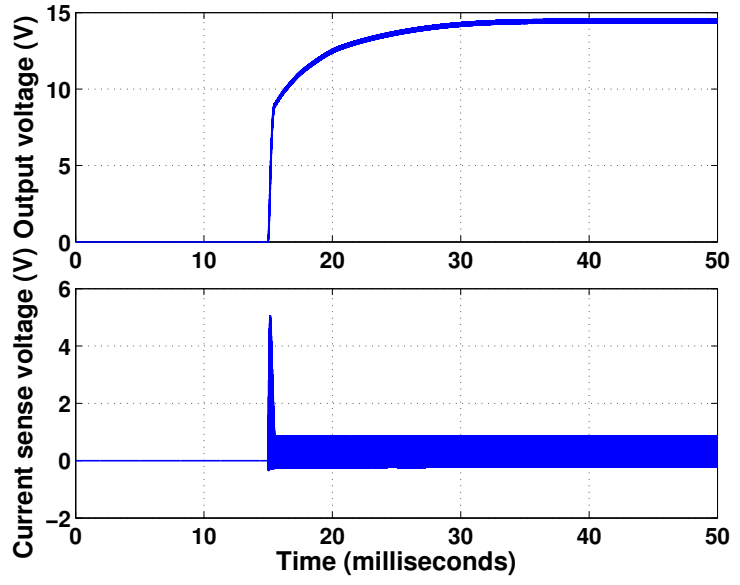

(a) Simplified model simulation results: $v_{\text {out }}=$ upper curve, $v_{c s}=$ lower curve

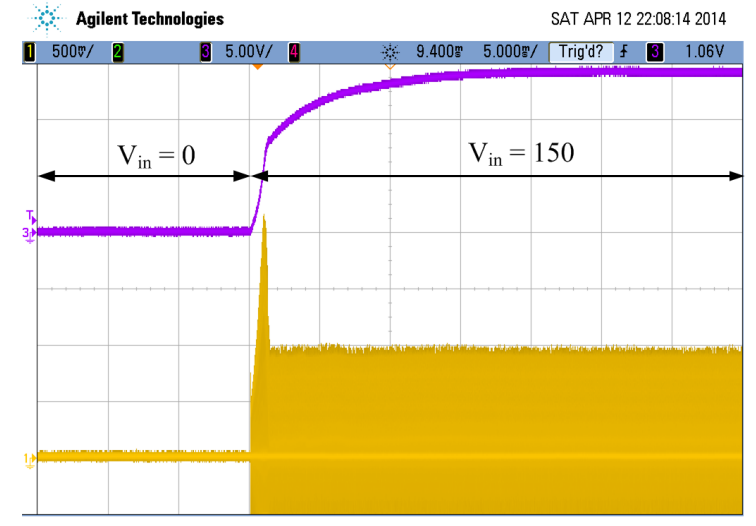

(b) Experimental results: $v_{\text {out }}=\mathrm{Ch} 3[5 \mathrm{~V} / \mathrm{div}], v_{c s}=\mathrm{Ch} 1$ $[500 \mathrm{mV} / \mathrm{div}]$

Fig. 5. Simulation and experimental output voltages and inductor currents (through current sense voltages) of the open-loop converter in response to a 0 to $150 \mathrm{~V}$ input voltage step, $R=6.9 \Omega, d=0.2$ and $f_{p w m}=80 \mathrm{kHz}$

as that of the intra-cycle responses. Two more tests are performed to verify the open-loop large-signal behaviour of the control-oriented model. In the first assessment, a voltage step $v_{i n}(t)$, from 0 to $150 \mathrm{~V}$, is applied to the input of the converter while the output load and PWM signal are unchanged and configured as $R=6.9 \Omega$, with a duty ratio $d=$ 0.2 and a switching frequency $f_{p w m}=80 \mathrm{kHz}$. The simulation and experimental open-loop transient responses are plotted in Fig. 5. For the second test, a $6.9 \Omega$ to $53.8 \Omega$ step load is effected at the output of the flyback converter, while setting the input voltage $V_{i n}=150 \mathrm{~V}$ and the PWM control signal with $d=0.1$ and $f_{p w m}=80 \mathrm{kHz}$. Fig. 6 illustrates the largesignal transient responses of the simulation and experimental output voltages from the second test. The results from both Figs. 5 and 6 confirm that the control-oriented model can generate an accurate inter-cycle transient response and is valid for large-signal verification.

\section{Application to a Digital CONTROL DESign}

Though the control-oriented model in Section II can not be used directly to synthesize a digital compensator, it permits to realistically verify the large-signal stability 


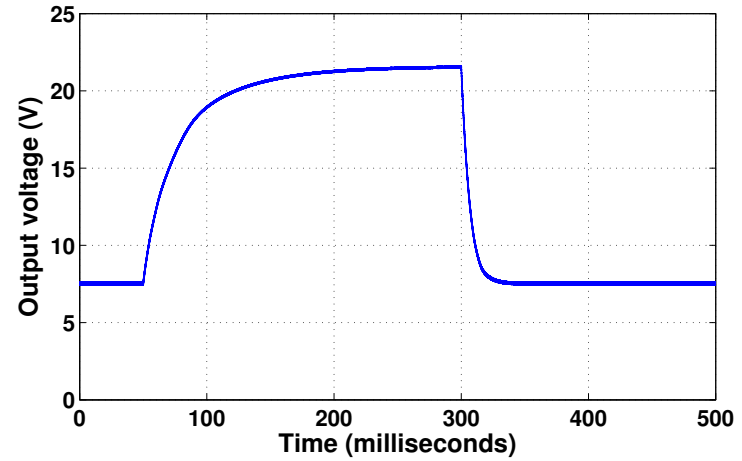

(a) Simplified model simulation results: $v_{\text {out }}$

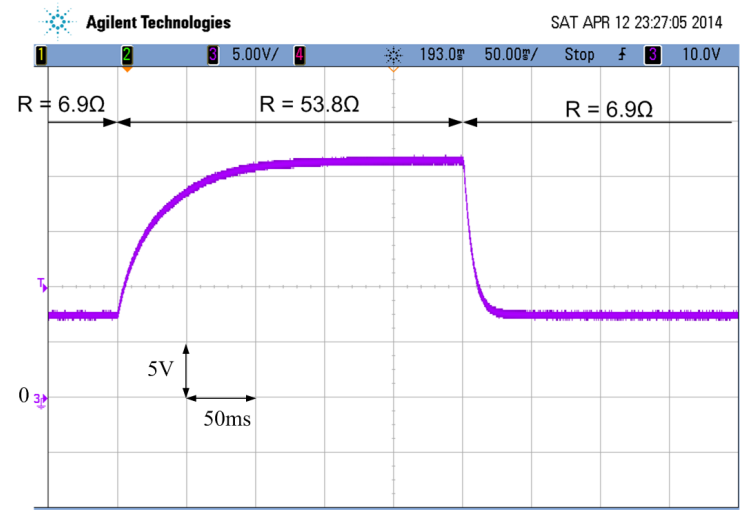

(b) Experimental results: $v_{\text {out }}=\mathrm{Ch} 3[5 \mathrm{~V} / \mathrm{div}]$.

Fig. 6. Simulation and experimental output voltages of the open-loop converter in response to a $6.9 \Omega$ to $53.8 \Omega$ step load, $V_{\text {in }}=150 \mathrm{~V}, d=0.1$ and $f_{p w m}=80 \mathrm{kHz}$

and performance of a closed-loop controlled converter. For demonstration, the simulation of the micro-controller-based, efficiency-optimized, PCM control for a flyback converter, as shown in Fig. 1, is considered. The operation principle of this control solution has been described in detail in [4] and so is not presented in here. In order to guarantee stability over wide operating range of the flyback converter and achieve adequate transient performance, a predictive functional compensator (PFC) is employed. The parameters of such controller can be found in [4].

A standard step-load test from $118.18 \Omega$ to $6.19 \Omega$ is applied at the converter output to examining the control performance. The transient response of the closed-loop system over different working conditions from both simulation and experiment are captured and plotted in Fig. 7. As can be seen from Fig. 7, the simulation results are consistent with the experimental ones and can accurately predict the behaviour of the closed-loop system under different loading conditions. The only inconsistency can be found is between the amplitude of the simulation and experimental current sense voltage. This discrepancy is due to the presence of the spike in the experiment inductor current waveform, which is typically ignored in the modelling process.

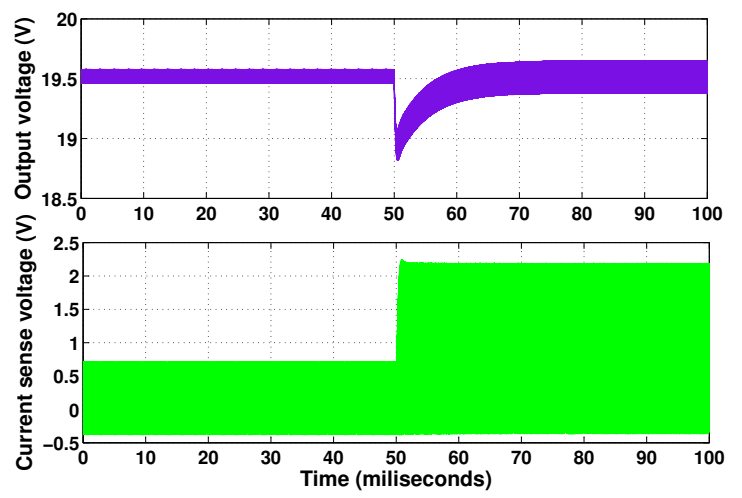

(a) Simplified model simulation results: $V_{\text {out }}=$ upper curve, $V_{c s}=$ lower curve

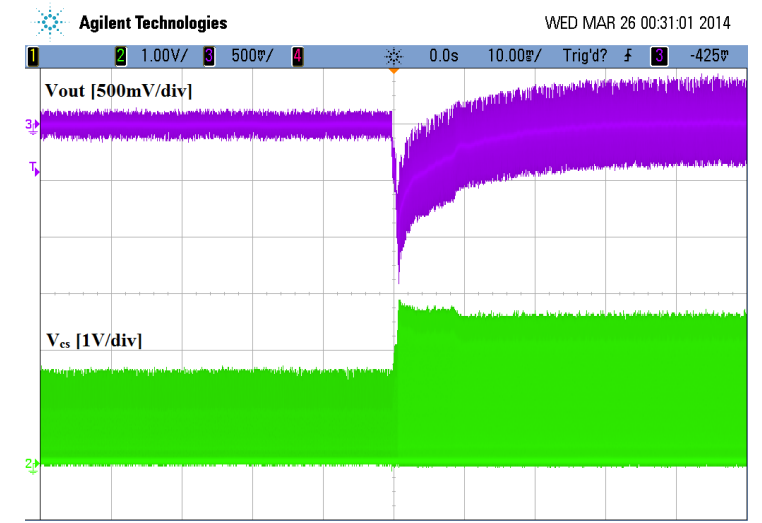

(b) Experimental results: $V_{\text {out }}=\mathrm{Ch} 3[500 \mathrm{mV} / \mathrm{div}], V_{c s}=\mathrm{Ch} 2$ [1 V/div]

Fig. 7. Simulation and experimental results of the converter output voltages and inductor currents (through current sense voltage) in response to a $118.18 \Omega$ to $6.19 \Omega$ step load with $V_{\text {in }}=150 \mathrm{~V}$

\section{CONCLUSION}

In this paper, a systematic approach was proposed which uses the knowledge of the previously determined full model in [5] as a starting point and simplifies this model to produce a control-oriented model. As pointed out in the paper, the model reduction process allows us not only to preserve the bulk of the model fidelity, which is critical for an effective control design phase, but also to significantly reduce the computational complexity. In addition to the model simplification, the equation-solving sequencing technique, which is used to break the algebraic loops and improve the simulation speed, is also discussed.

The proposed control-oriented model, in addition to providing a basis for control design itself, can serve as an effective platform for the evaluation of various closed-loop flyback control strategies. The results, from both simulation and experiment, confirm that the control-oriented model can achieve adequate intra-cycle dynamic fidelity, accurate intercycle responses and, most importantly, offers significant computational complexity reduction compared to the full modelling approach. 


\section{APPENDIX: STATE-SPACE MATRICES FOR VARIOUS SWITCH CONFIGURATION}

In brevity, the following definitions are used in the derivation of the state space matrices, $r_{Q w}=r_{Q o n}+r_{w}, n_{p s}=\frac{N_{s}}{N_{p}}$, $r_{D c}=r_{D o n}+r_{c}, r_{z d s}=\frac{r_{z} r_{d s}}{r_{z}+r_{d s}}$.

1) Configuration 1: $Q$ on, $D$ off and $D_{s}$ off:

$$
\begin{aligned}
A_{1} & =\left[\begin{array}{cccc}
0 & -\frac{r_{Q w}}{L_{l k}+L m} & 0 & 0 \\
0 & -\frac{r_{w}}{L_{l k}+L m} & 0 & 0 \\
0 & \frac{1}{C_{d s}} & -\frac{1}{r_{d s} C_{d s}} & 0 \\
0 & 0 & 0 & 0
\end{array}\right], \\
B_{1} & =\left[\begin{array}{cccc}
-\frac{1}{L_{l k}+L m} & 0 & 0 & 0 \\
-\frac{1}{L_{l k}+L m} & 0 & 0 & 0 \\
0 & 0 & 0 & 0 \\
0 & -\frac{1}{C} & 0 & 0
\end{array}\right], \\
C_{1} & =\left[\begin{array}{cccc}
0 & 1 & 0 & 0 \\
0 & -r_{Q w} & 0 & 0 \\
0 & 0 & 0 & 1
\end{array}\right], D_{1}=\left[\begin{array}{cccc}
0 & 0 & 0 & 0 \\
1 & 0 & 0 & 0 \\
0 & -r_{c} & 0 & 0
\end{array}\right],
\end{aligned}
$$

2) Configuration 2: $Q$ off, $D$ off and $D_{s}$ off:

$A_{2}=\left[\begin{array}{cccc}0 & -\frac{r_{Q w}+r_{d s}}{L_{k}+L m} & -\frac{1}{L_{l k}+L_{m}} & 0 \\ 0 & -\frac{r_{Q w}+r_{d s}}{L_{l k}+L m} & -\frac{1}{L_{l k}+L_{m}} & 0 \\ 0 & \frac{1}{C_{d s}} & 0 & 0 \\ 0 & 0 & 0 & 0\end{array}\right]$,

$B_{2}=\left[\begin{array}{cccc}-\frac{1}{L_{l k}+L m} & 0 & 0 & 0 \\ -\frac{1}{L_{l k}+L m} & 0 & 0 & 0 \\ 0 & 0 & 0 & 0 \\ 0 & -\frac{1}{C} & 0 & 0\end{array}\right], D_{2}=\left[\begin{array}{cccc}0 & 0 & 0 & 0 \\ 1 & 0 & 0 & 0 \\ 0 & -r_{c} & 0 & 0\end{array}\right]$,

$C_{2}=\left[\begin{array}{cccc}0 & 1 & 0 & 0 \\ 0 & -r_{Q w}-r_{d s} & -1 & 0 \\ 0 & 0 & 0 & 1\end{array}\right]$,

3) Configuration 3: $Q$ on, $D$ on and $D_{s}$ off:

$$
\begin{aligned}
A_{3} & =\left[\begin{array}{cccc}
\frac{r_{Q w}+\frac{r_{D c}}{n_{p s}}}{L_{l k}} & \frac{r_{D c}}{n_{p s}^{2} L_{l k}} & 0 & \frac{1}{n_{p s} L_{l k}} \\
\frac{r_{D c}}{n_{p s}^{2} L_{m}} & -\frac{r_{D c}}{n_{p s}^{2} L_{m}} & 0 & -\frac{1}{n_{p s} L_{m}} \\
0 & 0 & -\frac{1}{r_{d s} C_{d s}} & 0 \\
-\frac{1}{n_{p s} C} & \frac{1}{n_{p s} C} & 0 & 0
\end{array}\right], \\
B_{3} & =\left[\begin{array}{cccc}
\frac{1}{L_{l k}} & -\frac{r_{c}}{n_{p s} L_{l k}} & \frac{1}{n_{p s} L_{l k}} & 0 \\
0 & \frac{r_{c}}{n_{p s} L_{m}} & -\frac{1}{n_{p s} L_{m}} & 0 \\
0 & 0 & 0 & 0 \\
0 & -\frac{1}{C} & 0 & 0
\end{array}\right], \\
C_{3}= & {\left[\begin{array}{cccc}
1 & 0 & 0 & 0 \\
\frac{r_{D c}}{n_{p s}^{2}} & \frac{-r_{D c}}{n_{p s}^{2}} & 0 & \frac{-1}{n_{p s}} \\
\frac{-r_{c s}}{n_{p s}} & \frac{r}{n_{p s}} & 0 & 1
\end{array}\right], D_{3}=\left[\begin{array}{cccc}
0 & 0 & 0 & 0 \\
0 & \frac{r_{c}}{n_{p s}} & \frac{-1}{n_{p s}} & 0 \\
0 & -r_{c} & 0 & 0
\end{array}\right], }
\end{aligned}
$$

4) Configuration 4: $Q$ off, $D$ on and $D_{s}$ off:

$$
\begin{aligned}
A_{4} & =\left[\begin{array}{cccc}
-\frac{r_{Q w}+r_{d s}+\frac{r_{D c}}{n_{p s}^{2}}}{L_{l k}} & \frac{r_{D c}}{n_{p s}^{2} L_{l k}} & -\frac{1}{L_{l k}} & \frac{1}{n_{p s} L_{l k}} \\
\frac{r_{D c}}{n_{p s}^{2} L_{m}} & -\frac{r_{D c}}{n_{p s}^{2} L_{m}} & 0 & -\frac{1}{n_{p s} L_{m}} \\
\frac{1}{C_{d s}} & 0 & 0 & 0 \\
-\frac{1}{n_{p s} C} & \frac{1}{n_{p s} C} & 0 & 0
\end{array}\right], \\
B_{4} & =\left[\begin{array}{cccc}
\frac{1}{L_{l k}} & -\frac{r_{c}}{n_{p s} L_{l k}} & \frac{1}{n_{p s} L_{l k}} & 0 \\
0 & \frac{r_{c}}{n_{p s} L_{m}} & -\frac{1}{n_{p s} L_{m}} & 0 \\
0 & 0 & 0 & 0 \\
0 & -\frac{1}{C} & 0 & 0
\end{array}\right], \\
C_{4} & =\left[\begin{array}{cccc}
1 & 0 & 0 & 0 \\
\frac{r_{D c}}{n_{p s}^{2}} & \frac{-r_{D c}}{n_{p s}^{2}} & 0 & \frac{-1}{n_{p s}} \\
\frac{-r_{c}}{n_{p s}} & \frac{r_{c}}{n_{p s}} & 0 & 1
\end{array}\right], \quad D_{4}=\left[\begin{array}{cccc}
0 & 0 & 0 & 0 \\
0 & \frac{r_{c}}{n_{p s}} & \frac{-1}{n_{p s}} & 0 \\
0 & -r_{c} & 0 & 0
\end{array}\right],
\end{aligned}
$$

5) Configuration 5: $Q$ off, $D$ on and $D_{s}$ on:

$$
\begin{aligned}
A_{5} & =\left[\begin{array}{cccc}
-\frac{r_{Q w}+r_{z d s}+\frac{r_{D c}}{n_{p s}^{2}}}{n_{l k}} & \frac{r_{D c}}{n_{p s}^{2} L_{l k}} & -\frac{r_{z}}{\left(r_{z}+r_{d s}\right) L_{l k}} & \frac{1}{n_{p s} L_{l k}} \\
\frac{r_{D c}}{n_{p_{s}}^{2} L_{m}} & -\frac{r_{D c}}{n_{p s}^{2} L_{m}} & 0 & -\frac{1}{n_{p s} L_{m}} \\
\frac{r_{z}}{\left(r_{z}+r_{d s}\right) C_{d s}} & 0 & -\frac{1}{\left(r_{z}+r_{d s}\right) C_{d s}} & 0 \\
-\frac{1}{n_{p s} C} & \frac{1}{n_{p s} C} & 0 & 0
\end{array}\right], \\
B_{5} & =\left[\begin{array}{cccc}
\frac{r_{z}}{\left(r_{z}+r_{d s}\right) L_{l k}} & -\frac{r_{c}}{n_{p s} L_{l k}} & \frac{1}{n_{p s} L_{l k}} & -\frac{r_{d s}}{\left(r_{z}+r_{d s}\right) L_{l k}} \\
0 & \frac{r_{c}}{n_{p s} L_{m}} & -\frac{1}{n_{p s} L_{m}} & 0 \\
\frac{1}{\left(r_{z}+r_{d s}\right) C_{d s}} & 0 & 0 & \frac{1}{\left(r_{z}+r_{d s}\right) C_{d s}} \\
0 & -\frac{1}{C} & 0 & 0
\end{array}\right], \\
C_{5}= & {\left[\begin{array}{cccc}
\frac{r_{z}}{r_{z}+r_{d s}} & 0 & -\frac{1}{r_{z}+r_{d s}} & 0 \\
\frac{r_{D c}}{n_{p s}^{2}} & -\frac{r_{D c}}{n_{p s}^{2}} & 0 & -\frac{1}{n_{p s}} \\
-\frac{r_{c}}{n_{p s}} & \frac{r_{c}}{n_{p s}} & 0 & 1
\end{array}\right], } \\
D_{5}= & {\left[\begin{array}{cccc}
\frac{1}{r_{z}+r_{d s}} & 0 & 0 & \frac{1}{r_{z}+r_{d s}} \\
0 & \frac{r_{c}}{n_{p s}} & -\frac{1}{n_{p s}} & 0 \\
0 & -r_{c} & 0 & 0
\end{array}\right], }
\end{aligned}
$$

\section{REFERENCES}

[1] "ENERGY STAR program requirements for single voltage external AC-DC and AC-AC power supply."

[2] Y. Li and J. Zheng, "A low-cost adaptive multi-mode digital control solution maximizing AC/DC power supply efficiency," in 25th Ann. IEEE Applied Power Electronics Conf. and Expo., pp. 349-354, Feb. 2010.

[3] S. H. Kang, D. Maksimovic, and I. Cohen, "Efficiency optimization in digitally controlled flyback DC-DC converters over wide ranges of operating conditions," IEEE Trans. Power Electron., vol. 27, pp. 3734 3748, Aug. 2012.

[4] T. T. Vu, S. O'Driscoll, and J. V. Ringwood, "Computationally efficient fixed-parameter digital control of power coverters." Proc. Int. Symb. on Industrial Electron. (ISIE), 2014, accepted for publishcation.

[5] T. Vu, S. O'Driscoll, and J. Ringwood, "Nonlinear dynamic transformer time-domain identification for power converter applications," IEEE Trans. Power Electron., vol. 29, pp. 318-327, Jan. 2014.

[6] D. Maksimovic, A. Stankovic, V. Thottuvelil, and G. Verghese, "Modeling and simulation of power electronic converters," Proc. IEEE, vol. 89 pp. 898-912, Jun. 2001.

[7] W. H. Press, S. A. Teukolsky, W. T. Vetterling, and B. P. Flannery, Numerical Recipes in C: The Art of Scientific Computing. Cambridge University Press, 1992. 